Supporting information for:

\title{
Coassembly of tobacco mosaic virus coat proteins into nanotubes with uniform length and improved physical stability
}

\author{
Kun Zhou ${ }^{\dagger *}$, Sabine Eiben ${ }^{*}$, and Qiangbin Wang ${ }^{*+}$
}

${ }^{\dagger}$ Key Laboratory for Nano-Bio Interface, Division of Nanobiomedicine and $i$-Lab, Suzhou Institute of Nano-Tech and Nano-Bionics, Chinese Academy of Sciences, Suzhou 215123, China

${ }^{\ddagger}$ University of Chinese Academy of Sciences, Beijing 100049, China

${ }^{\S}$ Department of Molecular Biology and Plant Virology, Institute of Biomaterials and Biomolecular Systems, University of Stuttgart, Pfaffenwaldring 57, D-70569 Stuttgart, Germany

*To whom correspondence should be addressed: E-mail: sabine.eiben@bio.uni-stuttgart.de, E-mail: qbwang2008@ sinano.ac.cn. 


\section{EXPERIMENTAL SECTION}

Preparation of $\boldsymbol{E}$. coli-derived T103Ccp and T103C-T158Kcp. The T103Ccp expression plasmid has been constructed through standard polymerase chain reaction (PCR) and overlap extension PCR. ${ }^{1}$ The T103C-T158Kcp expression plasmid was constructed based on the T103Ccp gene sequence with an additional tyrosine to lysine mutation at the $\mathrm{C}$-terminus of the $\mathrm{cp}$ gene. The gene was amplified using the forward primer 5'-GAGATATACATATGAGCTATAGCAT-3' and the reverse primer 5'-GGAATTCTCATTTCGCCGGGCCGC-3' containing the mutation. Then the gene segment was cloned into the pET32a(+) vector (Novagen) at NdeI-EcoRI restriction sites using T4 DNA ligase and transformed into E. coli BL21 (DE3) (Novagen) after verification by sequencing. The expression strains were cultured in Luria-Bertani medium and induced for protein expression by the addition of isopropyl $\beta$-D-1-thiogalactopyranoside (IPTG) (1mM) for 12 hours.

The E. coli cells were harvested and lysed by sonication in lysis buffer $(20 \mathrm{mM}$ Tris-HCl, pH 7.2; $20 \mathrm{mM} \mathrm{NaCl}, 20 \mathrm{mM}$ EDTA) containing $15 \mathrm{mM}$ dithiothreitol (DTT). After centrifugation at $20000 \mathrm{~g}$ (Sorvall, rotor: F10S) for $30 \mathrm{~min}$ the supernatants were collected. The target proteins T103Ccp and T103C-T158Kcp were purified using ammonium sulfate fractionation and stored in dissociation buffer (50 $\mathrm{mM} \mathrm{Na} \mathrm{CO}_{3} / \mathrm{NaHCO}_{3}$, pH 9.5; $\left.20 \mathrm{mM} \mathrm{DTT}\right)$ at $-80{ }^{\circ} \mathrm{C}$. For the coassembly process, the protein solutions were thawed, dialyzed against $75 \mathrm{mM}$ sodium potassium phosphate buffer (SPP) pH 7.2 with $20 \mathrm{mM}$ DTT and stored at $4{ }^{\circ} \mathrm{C}$. 
Preparation of Plant-derived WTcp and T158KCcp. WT TMV and the mutant T158K TMV were prepared based on plasmid p843pe35TMVr. $1^{2}$ and the virion particles were isolated from the infected N. tabacum Samsun nn plants as previously described. $^{3}$ Purification of WTcp and T158Kcp was carried out using the acetic-acid-method as described by Fraenkel-Conrat. ${ }^{4}$

Preparation of TMV Genomic RNA. TMV genomic RNA was isolated from native TMV particles by means of phenol/chloroform extraction and alcohol precipitation: $800 \mu \mathrm{L}$ of virus suspension $(10 \mathrm{mg} / \mathrm{mL})$ were mixed with an equal volume of phenol : chloroform (1: 1) mixture, shook and centrifuged at $20000 \mathrm{~g}$ for 1 min. The supernatant was extracted two more times and then mixed with an equal volume of chloroform to remove residual phenol. After centrifugation, the RNA in the aqueous phase was precipitated by adding 1/10th volume of sodium acetate ( $\mathrm{pH}$ 5.2, 3 M) and 2.5 volumes of ethanol and incubation at $-20{ }^{\circ} \mathrm{C}$ for $15 \mathrm{~min}$. After centrifugation at $20000 \mathrm{~g}$ for $15 \mathrm{~min}$ at $4{ }^{\circ} \mathrm{C}$, the obtained pellet was rinsed with $70 \%$ cold ethanol. Carefully remove the supernatant and then dissolve the pellet gently in DEPC-treated water.

Coassembly of Two TMVcp Templated by TMV Genomic RNA. In general, different amounts of two TMVcp (final concentration: $1.3 \mathrm{mg} / \mathrm{ml}$ ) were mixed in SPP buffer (pH 7.2, $75 \mathrm{mM}$ ) containing $20 \mathrm{mM}$ DTT and incubated on ice for 6 hours to disassemble into oligo-subunits. Then, samples were incubated at room temperature overnight to form hybrid disks, followed by the addition of TMV genomic RNA (final concentration: $50 \mu \mathrm{g} / \mathrm{ml}$ ) which guides the disk assembly into nanotubes by 
incubation overnight at $30^{\circ} \mathrm{C}$.

pH Stability Test of Assembled Nanotubes. The freshly purified WTcp nanotubes and T103Ccp hybrid nanotubes were dialyzed against $\mathrm{pH} 7.0 \mathrm{SPP}$ buffer $(10 \mathrm{mM})$ at first. For DTT treated samples, the SPP buffer $(\mathrm{pH} 7.0,10 \mathrm{mM})$ containing $10 \mathrm{mM}$ DTT was used. Then, the $\mathrm{pH}$ value of the samples was adjusted to either 7.0, 9.5, 10, $10.5,11$, or 11.5 by adding high-concentration buffers of different $\mathrm{pH}$ values. The $\mathrm{pH}$ was controlled by $\mathrm{pH}$ meter (SevenCompact ${ }^{\mathrm{TM}} \mathrm{pH} /$ Ion, Mettle-Toledo, Giessen, Germany). For $\mathrm{pH}$ 9.5, 10 and 10.5, $\mathrm{Na}_{2} \mathrm{CO}_{3} / \mathrm{NaHCO}_{3}$ buffers were used. For $\mathrm{pH} 11$ and $11.5, \mathrm{Na}_{3} \mathrm{PO}_{4}$ buffers were used. The final protein concentration in all samples was $1 \mathrm{mg} / \mathrm{ml}$. After incubation at room temperature for 5 hours, equal amounts of samples were subjected to agarose gel electrophoresis. For densitometry analysis, the grayscale intensity of nanotube band was measured by the ImageJ software. The percentage of preserved, intact nanotubes was calculated by the grayscale intensity means of intact nanotube band in comparison to the control sample at $\mathrm{pH}$ 7.0.

Gel Electrophoretic Analysis. To determine the electrophoretic mobility of mixed nanotubes and for the stability investigation, samples were loaded onto $0.9 \%$ agarose gels in SPP buffer (pH 7.2, $50 \mathrm{mM}$ ). The electrophoresis was performed at $25 \mathrm{~V}$ and $25{ }^{\circ} \mathrm{C}$ for 18 hours. The protein in the cp nanotubes was stained with Coomassie Brilliant Blue R250 (Serva, Heidelberg, Germany). To analyze the retention factor (Rf) values, the migration distances of nanotube bands were measured and divided by the migration distance of the WTcp nanotube band.

For denaturing SDS-PAGE, samples were heated for $5 \mathrm{~min}$ at $100{ }^{\circ} \mathrm{C}$ in loading 
buffer (50 mM Tris-HCl, pH 6.8; $2 \%$ (w/v) SDS, 0.1\% (w/v) bromophenol blue, $10 \%$ glycerol) with or without $50 \mathrm{mM}$ DTT corresponding to reducing or nonreducing SDS-PAGE.

Isopycnic Density-Gradient Centrifugation. Samples of co-assembled nanotubes were mixed with $\mathrm{CsCl}$ in SPP buffer $(\mathrm{pH} 7.2,10 \mathrm{mM})$ to obtain an initial concentration of $33 \%(\mathrm{w} / \mathrm{w}) \mathrm{CsCl}$ and then centrifuged to isopycnic equilibrium using Sorvall TV 1665 Rotor (Thermo Scientific, Langenselbold, Germany) at 55000 rpm for 17 hours at $18{ }^{\circ} \mathrm{C}$. The distinct band of assembled nanotubes was collected, dialyzed against SPP buffer (pH 7.0, $10 \mathrm{mM}$ ) and examined by TEM.

Transmission Electron Microscopy (TEM). Samples (10 $\mu \mathrm{L})$ were adsorbed to carbon-coated copper grids, washed with $200 \mu \mathrm{L}$ of $\mathrm{ddH}_{2} \mathrm{O}$, and negatively stained for 2 min with $2 \%$ phosphotungstic acid. All samples were imaged on an FEI Tecnai 20 TEM operated at $200 \mathrm{kV}$. Images were recorded with a Gatan Ultra Scan 894 CCD camera and processed with the ImageJ software. 
Theoretical $\mathrm{pl}$ for TMVcp sequence

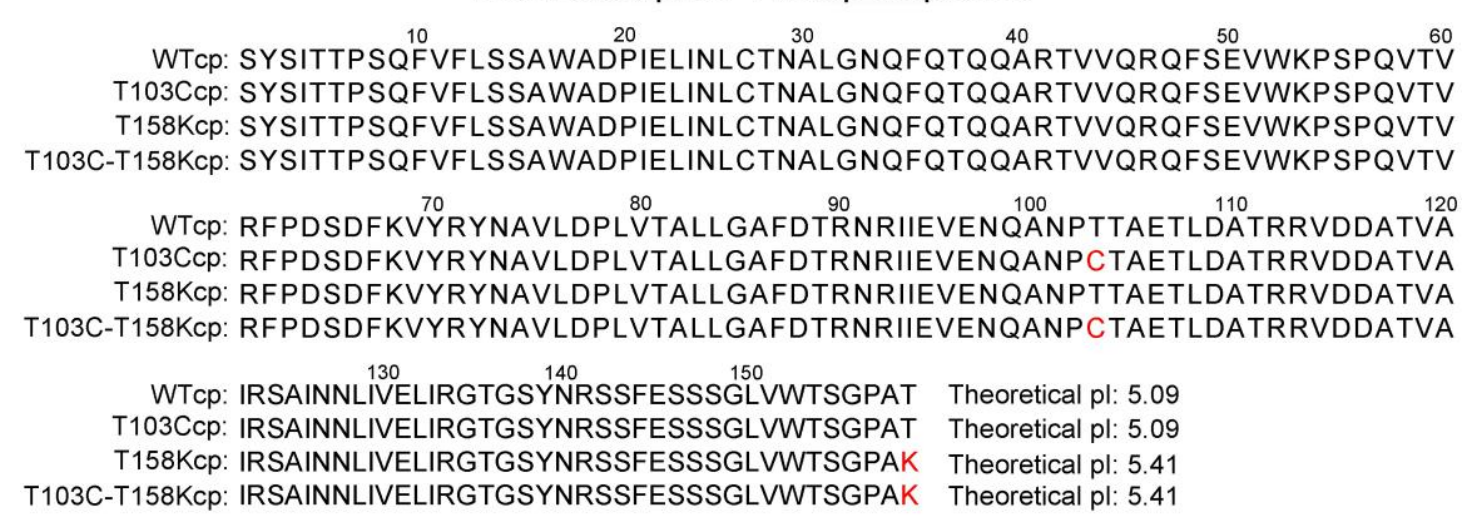

Figure S1. The theoretical isoelectric point (pI) of WTcp, T103Ccp, T158Kcp, and T103C-T158Kcp (calculated by http://web.expasy.org/compute_pi/). The red marks indicate the mutations. 


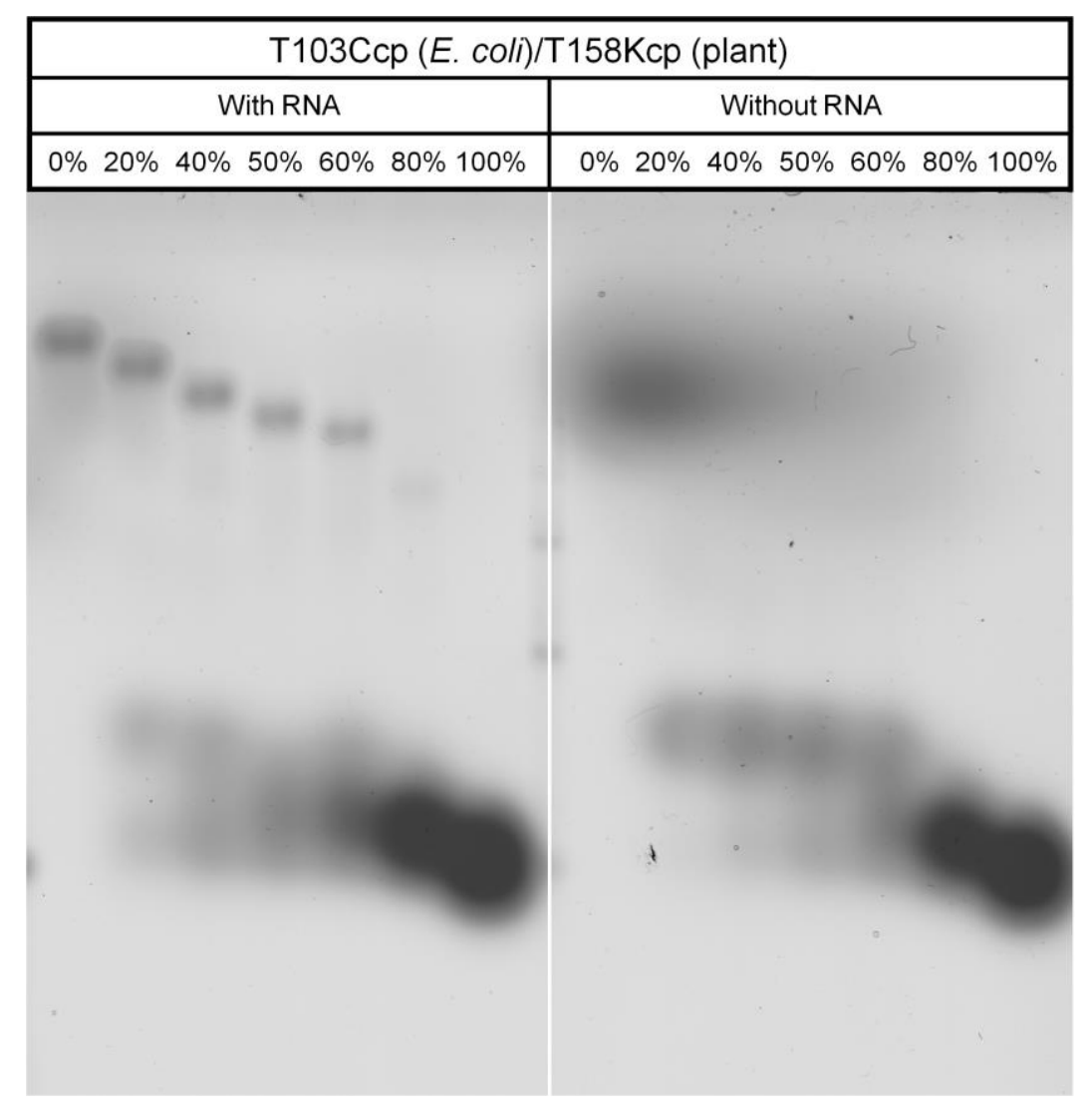

Figure S2. Native agarose gel electrophoresis of the co-assembled samples of $E$. coli-derived T103Ccp with plant-derived T158Kcp in the presence of TMV genomic RNA compared to the samples without RNA. RNA-templated packaging of T158Kcp and T103Ccp resulted in clearly defined bands, whereas only smears could be observed for cp mixtures in the absence of genomic RNA. 

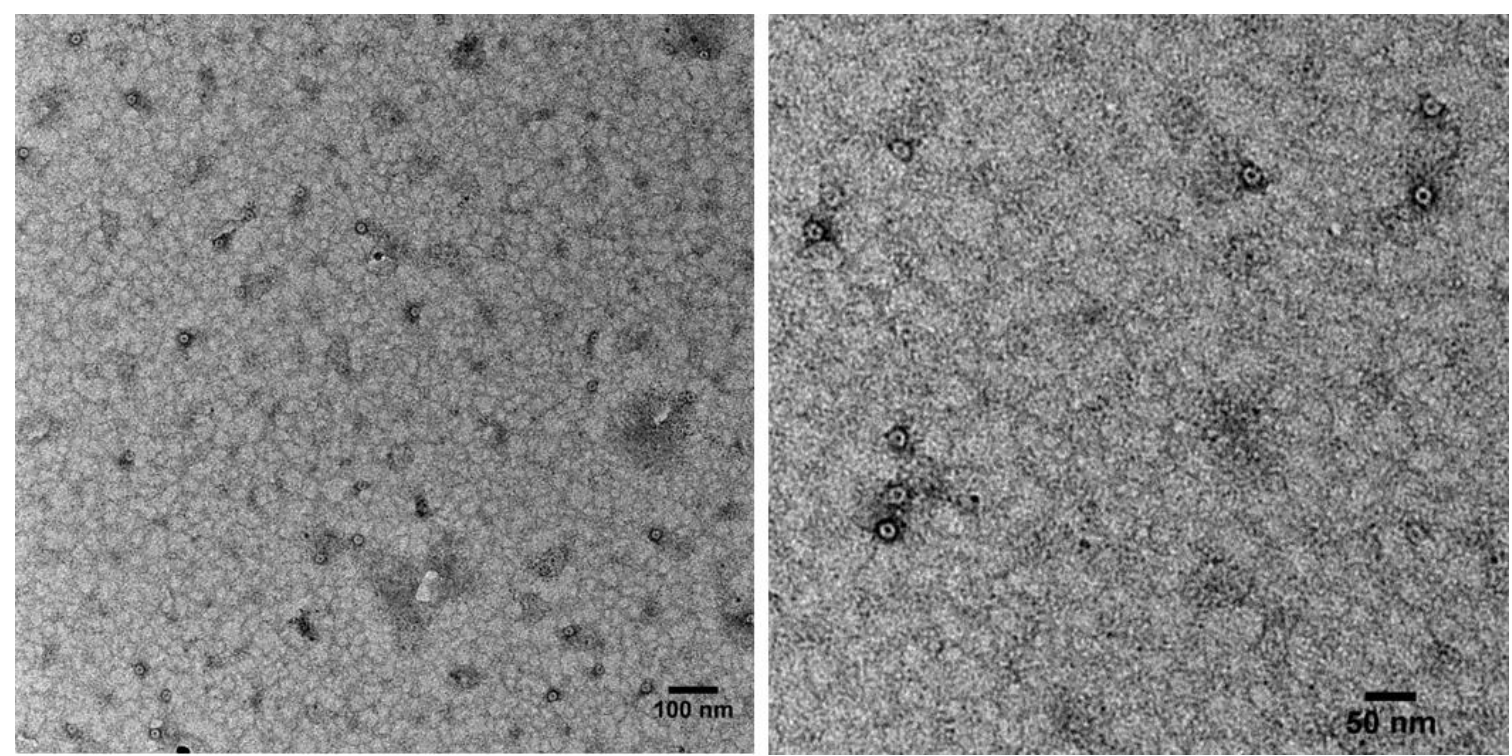

Figure S3. TEM images of the sample containing solely E. coli-derived T103Ccp and TMV genomic RNA. 


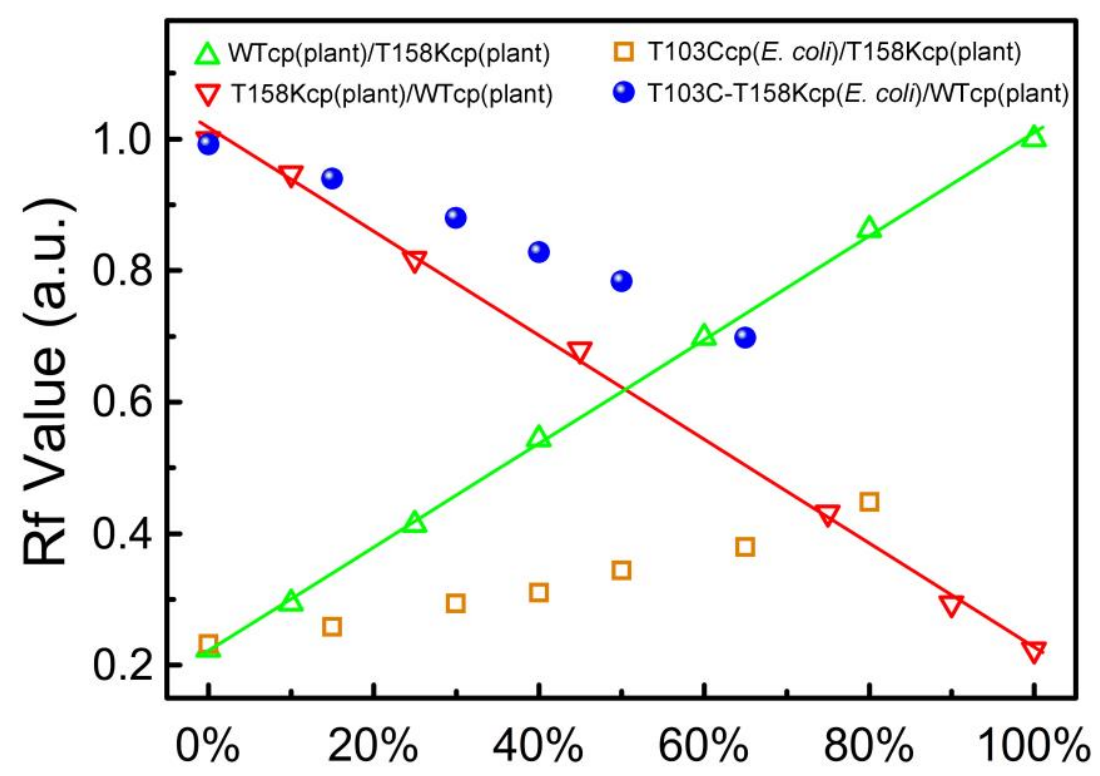

Figure S4. Comparison of the Rf values of defined nanotube bands composed of various TMVcp components at different ratios from $0 \%$ to $100 \%$. The mixed assembly of plant-derived WTcp and T158Kcp demonstrates well the linear relationship between $\mathrm{Rf}$ values of nanotube bands and the proportion of the proteins and provides an indicator for the incorporation rate of E. coli-derived TMVcp in the hybrid nanotubes. 


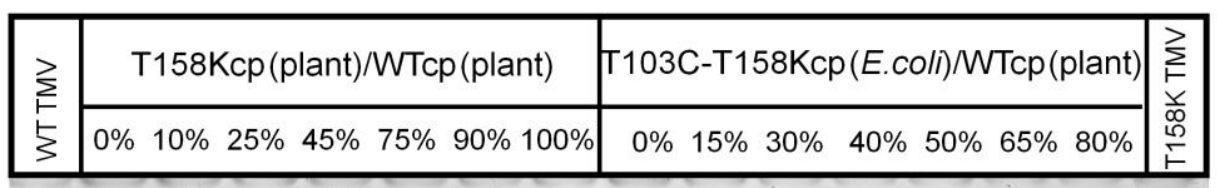

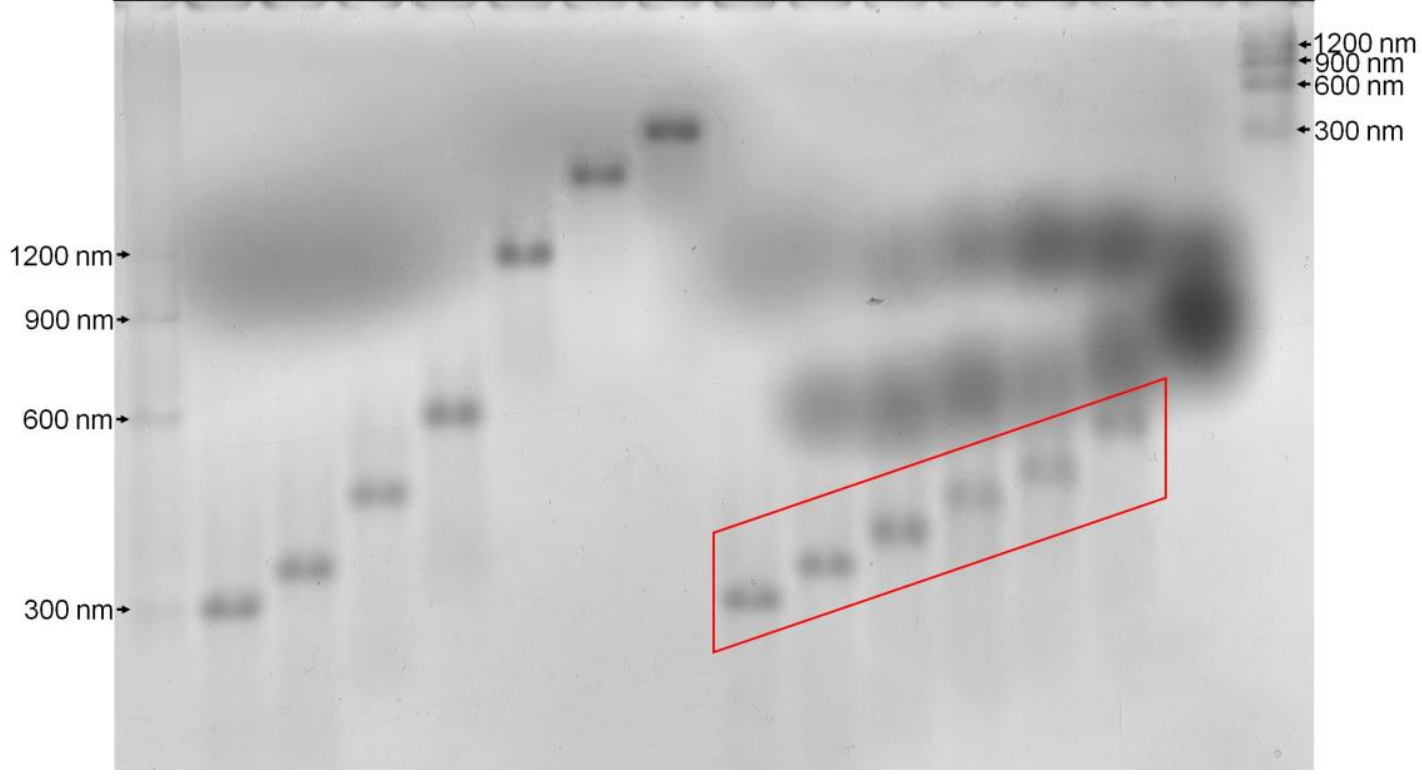

Figure S5. Native agarose gel of the co-assembled nanotubes of E. coli-derived T103C-T158Kcp and plant-derived T158Kcp mixed with plant-derived WTcp in the presence of TMV RNA. The increasing ratios of E. coli-derived T103C-T158Kcp or plant-derived T158Kcp (\%) in the mixture result in defined bands with gradually decreased electrophoretic mobility. The bands referring to TMV nanotubes composed of T103C-T158Kcp and WTcp are marked by the red frame. The two kinds of cloudy bands above the red frame correspond to residual T103C-T158Kcp/WTcp hybrid disks and pure T103C-T158Kcp aggregates/disks. As a reference, the WT and T158K particles isolated from $N$. tabacum were included leading to the bands corresponding to typical $300 \mathrm{~nm}$ nanotubes and head-to-tail multimers with lengths of $300 \mathrm{~nm}, 600$ $\mathrm{nm}, 900 \mathrm{~nm}$, and $1200 \mathrm{~nm}$, respectively. 


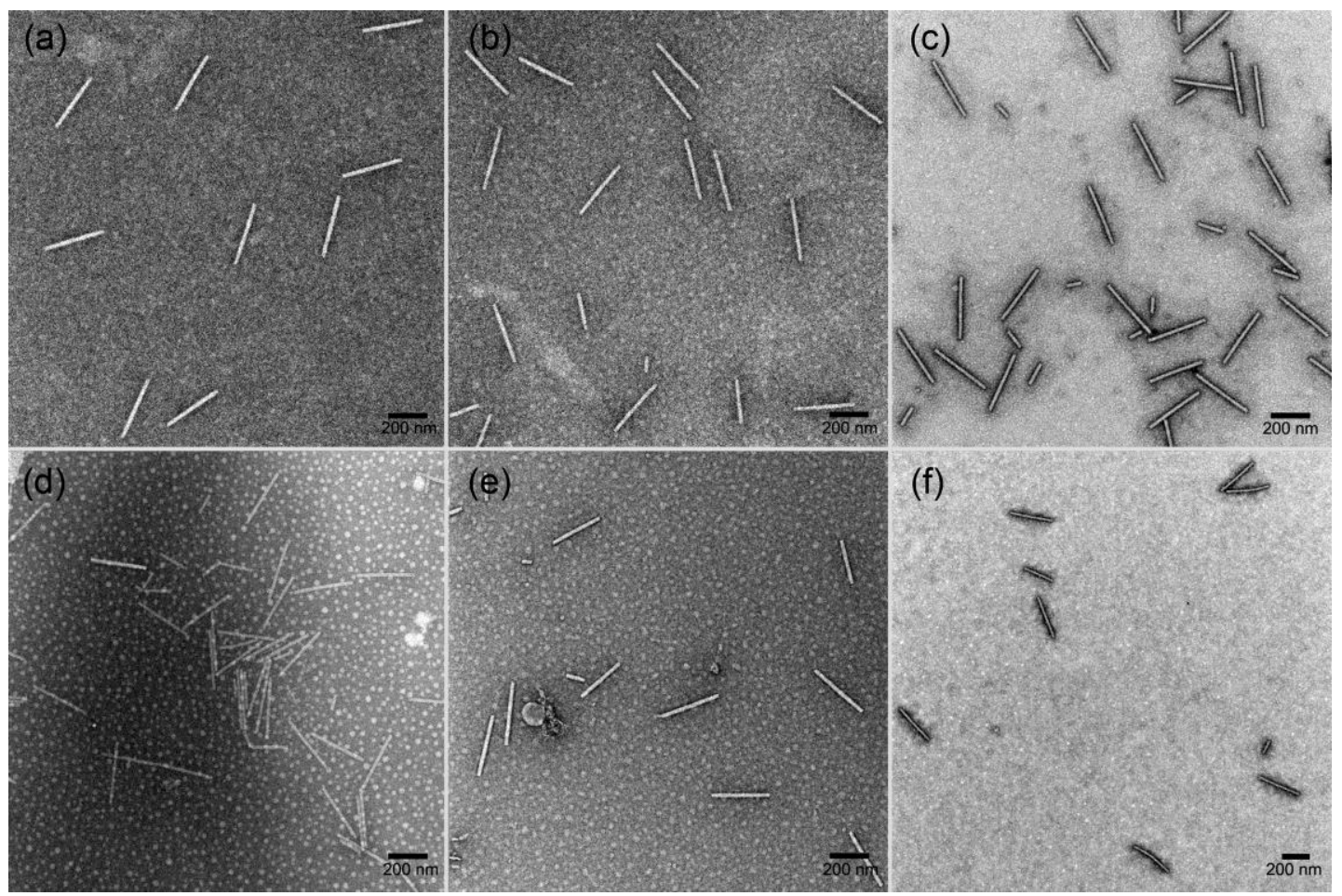

Figure S6. TEM images of the co-assembled samples with different amounts of $E$. coli-derived T103C-T158Kcp from $0 \%$ (a) to $15 \%$ (b), $30 \%$ (c), $40 \%$ (d), $50 \%$ (e), and $65 \%(f)$ in the mixtures. 


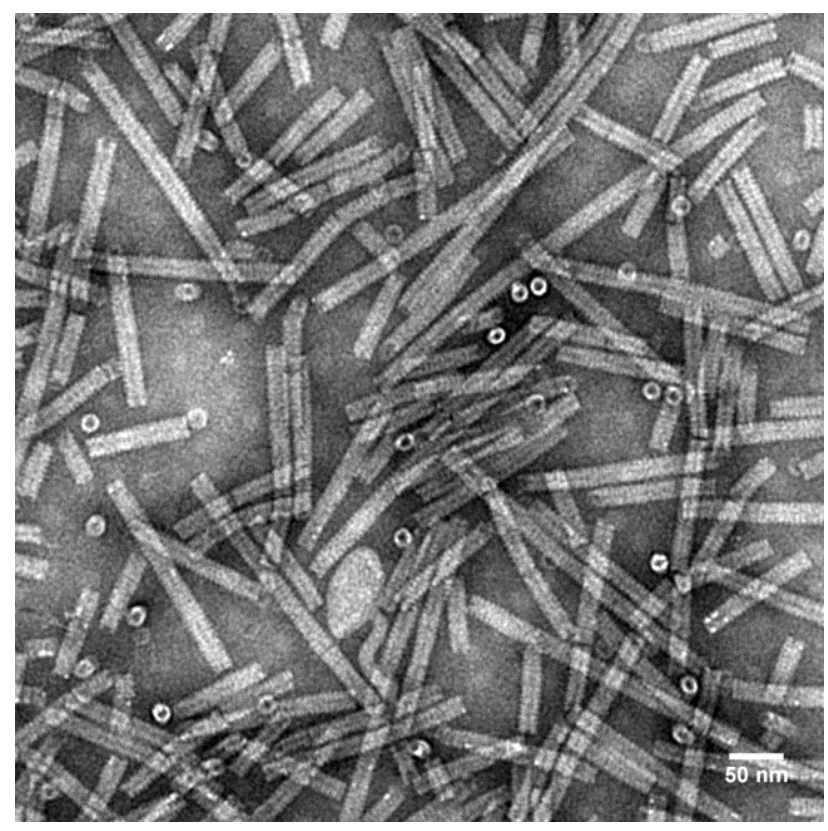

Figure S7. Purified T103C protein nanotubes. In the absence of genomic RNA, the TMV T103Ccp nanotubes are deficient in uniformity and programmability in length. 


\section{REFERENCES}

1. Zhou, K.; Li, F.; Dai, G. L.; Meng, C.; Wang, Q. B. Disulfide Bond: Dramatically Enhanced Assembly Capability and Structural Stability of Tobacco Mosaic Virus Nanorods. Biomacromolecules 2013, 14, 2593-2600.

2. Kadri, A. Maiss, E. Amsharov, N. Bittner, A. M. Balci, S. Kern, K. Jeske H. Wege, C. Engineered Tobacco Mosaic Virus Mutants with Distinct Physical Characteristics In Planta and Enhanced Metallization Properties. Virus Res. 2011, 157, 35-46.

3. Geiger, F. C.; Eber, F. J.; Eiben, S.; Mueller, A.; Jeske, H.; Spatz, J. P.; Wege, C. TMV Nanorods with Programmed Longitudinal Domains of Differently Addressable Coat Proteins. Nanoscale 2013, 5, 3808-3816.

4. Fraenkel-Conrat, H. Degradation of Tobacco Mosaic Virus with Acetic Acid. Virology 1957, $4,1-4$. 